\title{
Customized Design of Insoles for Pressure Relief via FEM
}

\author{
Lei YANG ${ }^{\text {a }}$, Xueqin WANG ${ }^{\text {a }, \mathrm{b}, 1}$, Qiannan Deng ${ }^{\mathrm{a}}$ \\ ${ }^{a}$ Zhejiang Sci-tech University, College of Textile and Engineering (International \\ Institute of Silk), Hangzhou, Zhejiang 310018, China \\ ${ }^{b}$ Zhejiang Silk and Fashion Culture Research Center, Hangzhou, Zhejiang 310018, \\ China
}

\begin{abstract}
A method to design the insoles for pressure relief based on the plantar pressure distribution characteristics by FEM (Finite Element Method), so as to realize the customized insole service for different individuals. By establishing a composite model of foot bones and soft tissues, human foot simulation was conducted to obtain the plantar pressure distribution data of latex, sponge, TPU and EVA flat insoles respectively; MATLAB was used to binarize the plantar pressure distribution map obtained from the simulation and extract the contour of the plantar pressure concentration area for the design of pressure relieving insoles, and the design of insoles for pressure relieving insoles was carried out according to the different properties of the four materials. The design of the insole combination is based on the different properties of the four materials. The appropriate solution of Insole is proposed by this design process as a prototype so as to achieve an intelligent approach for footwear design.
\end{abstract}

Keywords. Finite Element Method, Foot model, Personalized product customization, Pressure relief, Insole design

\section{Introduction}

With the development of computer technology and mathematical analysis, complex engineering issues can be obtained through a secondary tool such as computer. Therefore, numerical simulation technology has also become an effective means of solving engineering problems, which can shorten the design cycle of the product and reduce the development cost of the product. In recent years, the Finite Element Method(FEM) has been increasingly applied to the problem of foot mechanics mechanism, which can be analyzed by building a finite unit model of the object, obtaining all the stress and strain information inside the object, predicting the distributed load between the foot and the support, and also realizing the parametric evaluation of the material and shape of the shoe or insole[1], which has made a great help in the development stage of footwear products and greatly improved the efficiency. Since the foot characteristics of people are very different, and the functional insoles on the market are mostly designed for uniform groups, there is no way to meet the special needs of individuals for functional insoles, and customized insoles usually face the disadvantages of high customization cost and

${ }^{1}$ Xueqin WANG, Zhejiang Sci-tech University, No. 928, No. 2 street, Xiasha Higher Education Park, Hangzhou; E-mail:wxq780@163.com. 
long production period, therefore, using FEM, the personalized service of functional insoles can be customized for individuals.

\section{Related work}

There are more studies on the finite element simulation of the foot, and scholars such as a finite element digital model of the human foot was built by Yuning Zhou [2], including all bones, major cartilages, and ligaments, skin and soft tissues, inorder to lay the foundation for finite element mechanical analysis of the foot. A 3D finite element model of the human foot based on CT images was established Zhongkui Wang [3] and Wenxin Niu [4], who used reverse engineering ideas to establish a 3D finite element model of the human foot, and performed foot mechanics simulation, and verified the correctness of the model by comparing it with experimental date. A large amount of reference datas for foot mechanics simulation were provide by these studies. In the field of prevention and treatment of foot diseases, a new design method was proposed by Lei Tang for optimizing the stress distribution of the contact surfaces between the foot and the insole by applying functional gradient structural properties to the insole [5], which can effectively prevent foot ulcers. A kind of porous insoles to reduce the surface pressure of soft tissues was designed by Zijun Cao [6] based on the plantar pressure features of diabetic patients by finite element, which provided a reference for the design study of pressure-reducing insoles for diabetic patients. A method that constructed a finite element model by scaling a standard foot model had proposed an efficient and low-cost solution for personalized diabetic foot modeling and insole design to precisely reduce plantar pressure[7]. The stress effect of insoles with different structures and material stiffness on the foot was investigated by Hao-Wei Zhang through finite element simulations on the foot mechanics of patients with heel pain and flat feet with mid-foot arthritis [8,9].

In addition, many researches on orthopedic insole design based on FEM was studied by scholars. Such as effect of different sole forms (horizontal, semi-contact, full contact), insole thickness and insole materials on plantar pressure conditions. Studied the effect of hardness of insole material and support arch height on arch correction on plantar pressure distribution was studied by Shonglun Su [11] through experiments and finite element modeling. The effect of orthopedic insoles on plantar pressure distribution and correction of flat feet was studied by Jiani Yang [12] and Yankun Han [13]; A theory was pointed out by Taisheng Gong [14] that full contact insoles increase the contact area between the foot and the insole, which has been shown to be effective in preventing the recurrence of ulcers, both from theory and long-term clinical follow-up studies.

Most of the existing insoles in the market are designed according to the inherent human habits, lacking certain theoretical data support, while in the field of foot finite element biomechanics research, it also mostly stays in providing reference and research ideas for the design of insoles, and there are few cases of redesigning insoles according to the pressure distribution on the bottom of the foot. For example, the effect of changes in material properties on the performance of the insole base on the example of changes in the material properties of the insole was studied by Antonella [15] via simulation. The pressure distribution of different lattice structure insoles in three states (running, jumping, and walking) was analyze by Zolfagharian [16] base on a finite element model to make an optimized design to improve the pressure distribution on the footbed. A method proposed by Wen Huang [17] is that obtained plantar pressure data through finite element 
simulation and completed the initial establishment of a customized insole model, and then optimized the design of the insole using Laplace deformation technique to redistribute plantar pressure and improve the phenomenon of local over-concentration of plantar pressure. The designed method proposed by Davie [18] is that sampled the foot pressure map, mapped the data points onto the insole surface, used the interplay of different spatial structures of equivalent materials, and then adjusted the density morphology of their spatial structures to obtain the final insole model using 3D printing.

\section{Design method}

In this paper, personalized insole customization service was realized through foot finite element simulation, in order to simplify the tedious steps of traditional insole customization and improve efficiency. Two materials with good pressure-relief effect, latex and sponge, were selected as pressure-relief shims. Their stress-strain data were measured by compression, tension and shear experiments and fitted with simulation data to verify that the simulation experiments of latex and sponge are effective, so that the next step of finite element simulation of foot-insole composite model can be carried out. And EVA and TPU as rigid insole materials can play a good role in arch support. Next, the mechanical properties of several materials were analyzed and compared respectively, and the mechanical action characteristics of the four materials on the foot were analyzed by simulation.

\subsection{Mechanical properties and simulation of latex and sponge}

\subsubsection{Microstructure of latex}

In addition, the study of the microstructure of latex was important for the analysis of the mechanical properties of latex. The microstructure of the latex was analyzed by electron microscopy scanning, focusing on the size of the micropores of the latex and calculating the porosity. The electron microscopy results were shown in Figure 1, and by using the scale, the diameter of one micropore in the figure can be calculated as $382.4 \mathrm{um}$.



Figure 1. Electron microscope scan analysis diagram

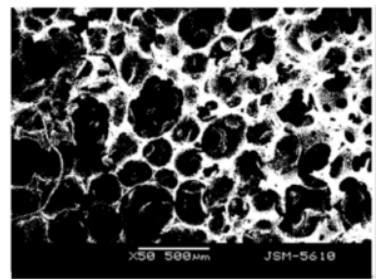

Figure 2. two-dimensional electron micrograph 
In order to find the porosity of the latex, the electron microscope scan was modulated into a two-dimensional figure by Adobe Photoshop software, as shown in Figure 2. The figure was imported into Image $\mathrm{J}$ software, and now the electron microscope scan was labeled with the length $(2535.71 \mathrm{um})$ and width $(1646.83 \mathrm{um})$ of the figure according to the scale, and the size of the whole figure was $4175883.3 \mathrm{um}^{2}$, and the pore area was $3001259.763 \mathrm{um}^{2}$, as shown in Figure 3 . The calculated porosity of latex is $72 \%$.

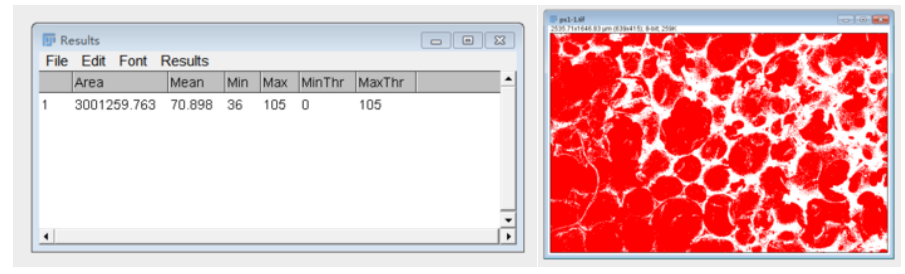

Figure 3. Porosity solving.

\subsubsection{Simulation analysis of the mechanical properties of latex and sponge}

The low-density foam simulation method was used to simulate the latex, and the obtained stress-strain curve was fitted and analyzed with the uniaxial compression experimental curve to compare the coefficient of determination $\mathrm{R}^{2}$, as shown in Figure 4, the $\mathrm{R}^{2}$ of the low-density foam simulation method was 0.9871 , and its fit was above $95 \%$, which was an excellent fit. The experiment proved that the compression experimental simulation based on the finite element method is effective and feasible.

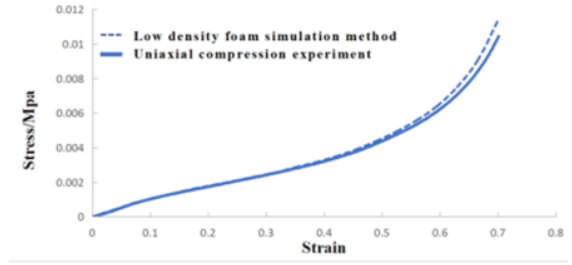

Figure 4. Comparison of compression simulation and experimental values(Latex).

The same low-density foam shear simulation method was used to simulate the experimental stress-strain curve, and the shear experimental curve was fitted and analyzed to compare the coefficient of determination $\mathrm{R}^{2}$. as shown in Figure 5, the $\mathrm{R}^{2}$ of the low-density foam shear simulation method was 0.9832 . the fit was above $95 \%$, and the fit was excellent. The experiment proved that the shear simulation based on the finite element method is effective and feasible.

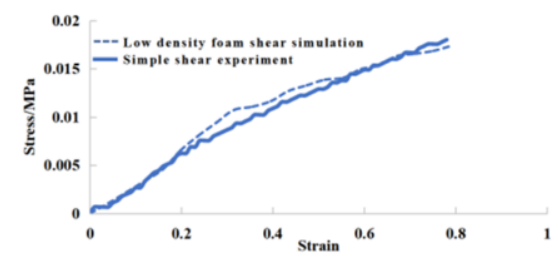

Figure 5. Comparison of shear simulation and experimental values(Sponge).

Referring to the method of latex to compare the sponge compression simulation results with the sponge compression test average, as shown in Figure 6, the results of the 
sponge foam simulation with the low-density foam method and the compression experimental average curve basically matched, the fit coefficient $\mathrm{R}^{2}$ of the two reached $99.9 \%$, the error was less than $0.1 \%$, the accuracy was high. Therefore, it was accurate and effective to use this method in the subsequent sponge simulation.

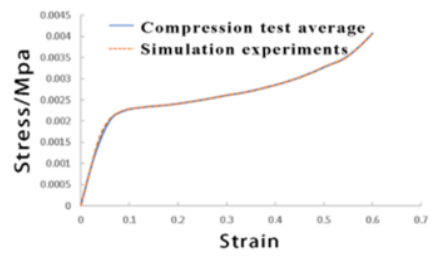

Figure 6. Comparison of compression simulation and experimental values（Sponge）.

\subsection{Finite element modeling of foot-insoles}

Abaqus is a powerful suite of finite element software for engineering simulation, with problem solving ranging from relatively simple linear analysis to many complex nonlinear problems. It has a library of various types of material models to simulate the properties of typical engineering materials, including metals, rubbers, polymers, composites, and more. In the field of CAE engineering, HyperMesh is best known for its powerful finite element meshing pre-processing capabilities. HyperMesh has excellent speed, adaptability and customizability in terms of efficiency and quality in processing geometric models and finite element meshes. Therefore, HyperMesh is chosen as the meshing software for the foot skeleton model and insole model, and then Abaqus is used to solve the model, analyze the mechanical characteristics of human foot, and achieve the optimal design of insole based on the sole pressure.

\subsection{1. foot composite model meshing on Hypermesh}

Intercepted the standard human foot model in C4D, retained the two parts of the skeleton and the foot shell, as shown in Figure 7, and then the model was imported into Hypermesh for pre-processing. Firstly, the area of interference between the models was processed, and the mesh size was set at about $10-20 \mathrm{~mm}$, and the sole part of the foot will be in contact with the insole, so the mesh density was smaller, and the mesh size of other areas was larger, which will reduce the overall mesh size and speed up the computing time. Then, the 2D mesh quality was checked, and modify the meshes with poor quality, and finally the solid cells were generated, and the final number of meshes generated for the skeletal part is 21649 and for the soft tissue part is 30502 . The foot model with the mesh divided in Hypermesh was shown in Figure 8.

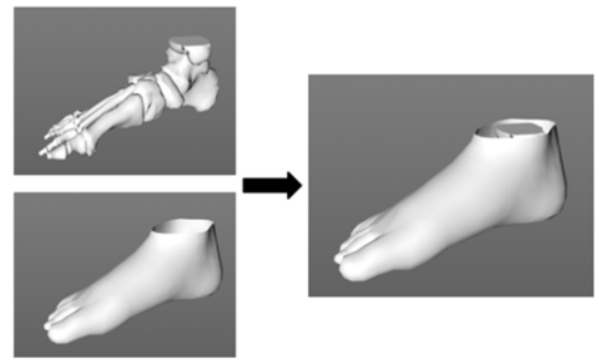

Figure 7. Foot model 


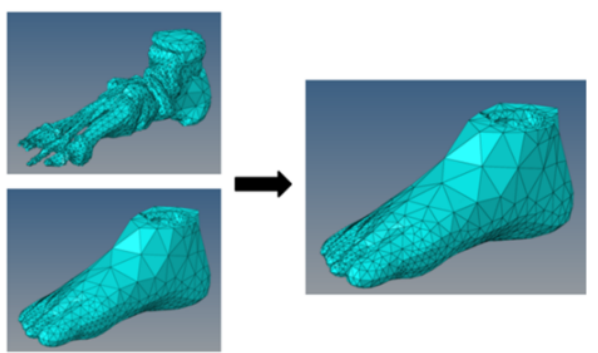

Figure 8. Meshed model

\subsubsection{Assembly of foot-insole composite models on abaqus}

Imported the model processed in Hypermesh into Abaqus, created a flat plate $(300 \mathrm{~mm} \times$ $150 \mathrm{~mm} \times 10 \mathrm{~mm})$ at a distance of $1-2 \mathrm{~mm}$ from the bottom of the foot, set the mesh size to $5 \mathrm{~mm}$, adjusted the position of the foot model, try to maintain a level with the flat plate, the assembly was complete, as shown in Figure 9.

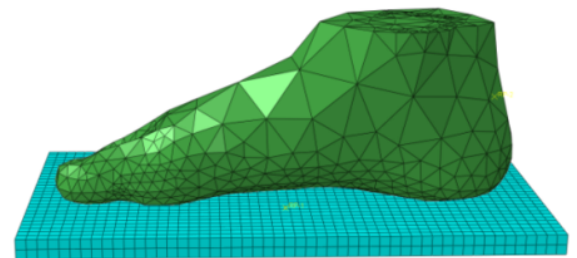

Figure 9. The completed foot-insole model assembled in Abaqus.

- Setting of material properties. The material properties of the insole were established in Abaqus, and the parameters of each part of the material were shown in Table 1, where latex and sponge are Low Density Foam, and only the stress-strain data obtained from the experiment can be imported, without setting the Young's modulus and Poisson's ratio.

- Contact setup. Selected General contact (Explicit) for the contact type. Specify that the upper surface of the insole made contact with the lower surface of the footbed, which reduced the amount of software operations. The contact between the insole and the soft tissues of the sole was defined as friction contact with a coefficient of friction of 0.6 .

- Setting of Load and boundary condition. When a person stands normally, his or her weight is shared by both feet. The weight of the experimenter selected for this paper is $54 \mathrm{~kg}$ and the foot length is $23 \mathrm{~cm}$, so about $270 \mathrm{~N}$ of gravity was bore by the right foot. In addition, the foot should also be subjected to $75 \%$ of the gravitational load of the Achilles tendon force. In order to simulate the standing condition, a Ground Reaction Force (GRF) of $270 \mathrm{~N}$ was applied at the center of the ground support and a vertical upward concentrated force of 202.5 N was applied at the Achilles tendon connection of the heel bone; the boundary condition was set to the distal fixation of the upper surface of the foot [9], as shown in Figure 10. 
Table 1. Parameter settings for various materials in Abaqus

\begin{tabular}{lccr}
\hline & Young's modulus/Mpa & Poisson's ratio & Density $\left(\mathrm{t} / \mathbf{m ~ m}^{\mathbf{3}}\right)$ \\
\hline Bone & 7300 & 0.3 & $2.3 \times 10^{-9}$ \\
Soft Tissue & 0.45 & 0.49 & $1.06 \times 10^{-9}$ \\
Latex & - & - & $7.3 \times 10^{-11}$ \\
Sponge & - & - & $5.3 \times 10^{-11}$ \\
TPU & 11.7 & 0.45 & $1.2 \times 10^{-9}$ \\
EVA & 2 & 0.35 & $9.29 \times 10^{-10}$ \\
\hline
\end{tabular}

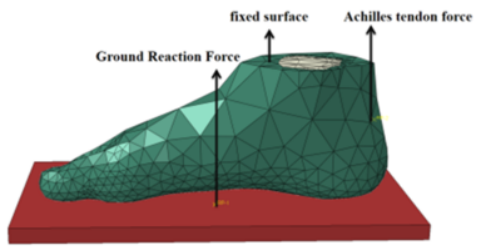

Figure 10. The completed foot-insole model assembled in Abaqus.

\subsection{Finite element simulation analysis of insoles of different materials}

As shown in Figure 11, the simulation results of the four materials, from left to right, are the cloud map of the plantar pressure distribution of latex, sponge, EVA and TPU. shows the peak stress data of different regions of the foot were shown in Figure 12, according to the analysis of the figure, it can be seen that the plantar pressure of TPU insoles was significantly higher than the other three materials, while latex, sponge and EVA three materials, the peak stress difference in the heel area was not significant, but in the forefoot area and toe area, latex pressure was the smallest, EVA was the largest. in summary, in the pressure distribution of various regions of the foot, latex material insoles pressure-relief effect was better than the other three materials.

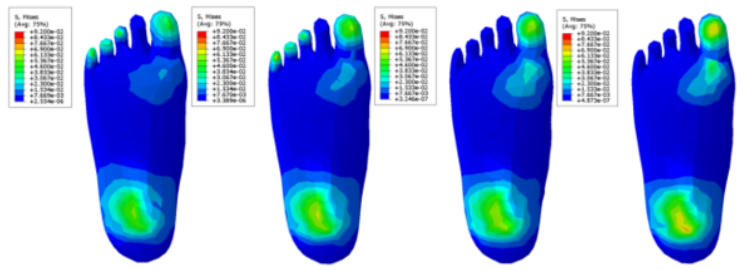

Figure 11. Cloud map of plantar pressure distribution of 4 materials.

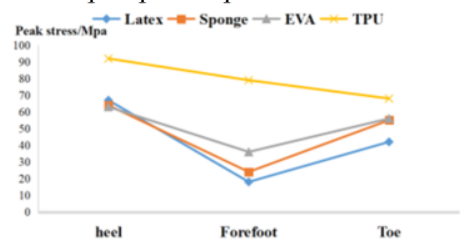

Figure 12. Stress peaks of 4 materials in different plantar regions.

\subsection{Footwear insole design process based on plantar pressure distribution characteristics}

According to the pressure distribution diagram of the footbed obtained from the simulation experiment, the pressure was more concentrated in the heel and forefoot area, 
so the latex material with better pressure-relief effect was added in these two areas. The foot contour from the human arch to the heel was extracted, and the arch full contact insole was designed. The materia TPU with high hardness, which can play a good role in supporting the arch and reducing the force on the heel by increasing the contact area. EVA was chose as The upper layer of the insole and the contact part of the sole.

Because EVA was the material in direct contact with the sole of the foot, the cloud map of the plantar pressure distribution of EVA was selected, and the image binarization process was carried out in MATLAB to convert the RGB image into a binarized image, so as to obtain the edge of the concentrated region of the plantar pressure, and then the design of latex pressure-relief insole was carried out according to the binarized image, as shown in Figure 13. The effect of the insole for pressure relief based on the pressure distribution characteristics of the footbed was shown in Figure 14. The material composition of each part of the insole were shown in Figure 15, part 1 is the EVA layer that fits the footbed, part 2 is the TPU arch support spacer that completely fits the arch and the footbed, and part 3 is the latex pressure relief spacer for the forefoot and the footbed.
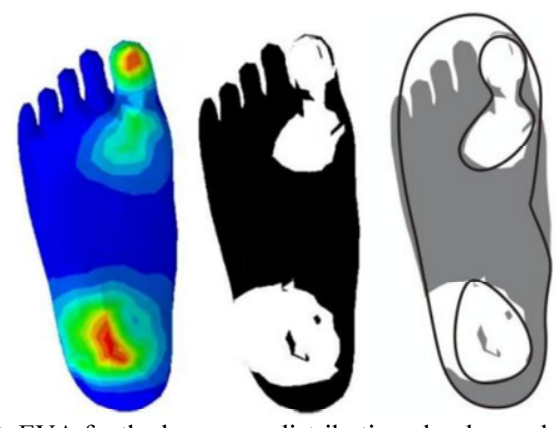

Figure 13. From left to right, EVA footbed pressure distribution cloud map, binarized image, insole pressure relief shim profile.

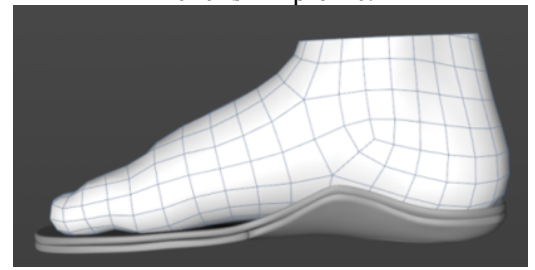

Figure 14. Shoe insole effect

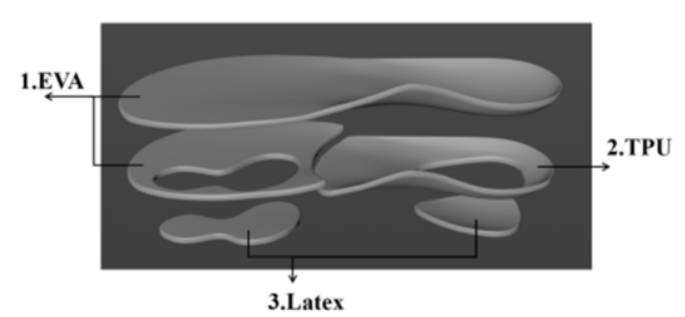

Figure 15. Diagram of the material composition of each part of the insole 


\section{Discussion}

A finite element model of the foot (bone + soft tissue) was built in this paper, the foot ligaments, plantar tendon membrane and other tissues were omitted, and the skeletal part was retained, which can reduce the difficulty of modeling and at the same time more realistic response to the mechanical characteristics of the foot when the human body is standing. Hypermesh was used to mesh the complex model, and the calculation was solved by Abaqus to obtain the plantar pressure distribution maps of four flat materials: latex, sponge, TPU and EVA. Based on the analysis of the results, it can be seen that the maximum pressure of the plantar area was generally found at the heel, and the pressure of TPU was the maximum in all areas of the plantar area, which were $92 \mathrm{kpa}$ in the rear foot area, $79 \mathrm{kpa}$ in the forefoot area, and $68 \mathrm{kpa}$ in the toe area, respectively. TPU was stiffer, has better support, and was suitable as an arch support material. The pressure of latex in the forefoot and toe area were less than the sponge, the pressure of two materials in the forefoot area were $18 \mathrm{kpa}$ and $24 \mathrm{kpa}$, the pressure of toe area was $42 \mathrm{kpa}$ and $55 \mathrm{kpa}$. therefore, the pressure-relief of latex on performance was slightly better than the sponge, suitable for the plantar pressure concentration area of the pressure-relief piece; and the pressure of EVA was between the four materials, so it was suitable for direct contact with the bottom of the foot material.

Based on the plantar pressure distribution map obtained from the above simulation experiment, the insole was optimally designed to realize the personalized customization service of the insole. The plantar pressure distribution map of EVA was selected and binarized in Matlab to obtain the contour of the plantar pressure concentration area, according to which the design of the pressure-relief shim was completed; since the contour of each person's foot is different, the contour of the foot model from the arch to the hindfoot area was extracted to design the arch support piece, and the material was selected from TPU; while the area in contact with the sole was selected from EVA, thus the customized design process of insoles based on plantar pressure was completed.

\subsection{Limitations}

In the process of foot model and insole assembly, it was difficult to find the relative equilibrium position of the foot model and insole model; in addition, if the conditions allow, increasing the number of meshes of the model can also make the simulation results closer to the real value.

\subsection{Future Work}

- The final designed insole will be simulated by FEM to obtain the pressure distribution map of the footbed and compared with the pressure of the flat insole to verify the designed insole has the function of pressure relief.

- The finished insole will be made by using 3D printing, and the experimenter who meets the simulation requirements is searched for to perform plantar pressure measurement and compare with the simulation results to verify the validity of the model and prove the feasibility of the personalized custom design method of the insole. 


\section{Conclusion}

The mechanical characteristics of human foot was analyzed in the paper through finite element simulation, and the influence of different insole materials on the plantar pressure distribution was studied, A method was proposed to optimize the design of insoles based on the plantar pressure distribution characteristics, so as to obtain the best design of pressure-relieving insoles and realize the customized service of insoles for individuals.

\section{Acknowledgement}

Thanks to support from National Natural Science Foundation of China (22075252) and National innovation program for college students(202110338001X).

\section{References}

[1] Liu J. Establishment of a foot-shoe finite element model and application in the study of the plantar fascia. Beijing Sport University. 2019.

[2] Zhou Y, Zhang H, Chen X, Yan Z, Liu F, Wang S. Establishment of three-dimensional finite element digital foot model. Chinese Journal of Tissue Engineering Research. 2015;19(05):662-666.

[3] Wang Z, Imai K, Kido M, et al. A finite element model of flatfoot (pes planus) for improving surgical plan. 2014 36th Annual International Conference of the IEEE Engineering in Medicine and Biology Society. 2014;844-847.

[4] Niu W, Yang Y, Yu G, Ding Z. Valid Constructing Method of Three-dimensional Finite Element Human Foot Modeland Experimental Analysis on Its Rationality. Journal of Biomedical Engineering. 2009;26(01):80-84.

[5] Tang L, Wang L, Bao W, et al. Functional gradient structural design of customized diabetic insoles. Journal of the Mechanical Behavior of Biomedical Materials. 2019;94(279-287).

[6] Cao Z, Wang F, He Y, et al. Plantar Pressure and Offloading Insole Structure for Diabetic Patients: A Finite Element Analysis. Chinese Journal of Rehabilitation Theory and Practice. 2021;27(07): 852-858.

[7] Zhu S, Li D, Tang L, et al.Design of Three-Dimensional Printed Diabetic Insoles with Gradient Modulus. Journal of Medical Biomechanics. 2021;36(01):102-109.

[8] Zhang H, Li L, Liu Y, Ren X. Biomechanical Study of Personalized Buffer Insoles for Heel Pain. Journal of Medical Biomechanics.2018;33(01):30-36.

[9] Zhang H, Yang J, Liu Y, Chen L. Biomechanical Study of Orthotic Insoles for Flatfoot Patients with Midfoot Arthritis.Chinese Journal of Biomedical Engineering. 2020;39(03):327-334.

[10] Steven G, Ahmet E, Marc P, et al. Reduction of plantar heel pressures: Insole design using finite element analysis. Journal of biomechanics, 2006;39(13): 2363-2370.

[11] Su S, Mo Z, Guo J, et al. The effect of arch height and material hardness of personalized insole on correction and tissues of flatfoot. Journal of healthcare engineering. 2017.

[12] Yang J, He H. Study of orthopedic insoles for the correction of flat feet in children aged 9-10 years. CHINA SPORT SCIENCE SOCIETY. 2019;2.

[13] Han Y, Huo H. A study on foot shape and gait characteristics of patients with flat foot. Chinese Journal of Rehabilitation Medicine. 2020;35 (4): 434-439.

[14] Gong T, Xu L, Tang Y. Research Progress on Total Contact Insoles at Home and Abroad. China Leather. 2013;42(20):112-114+119.

[15] Forestiero A, Raumer A, Carniel E L, et al. Investigation of the interaction phenomena between foot and insole by means of a numerical approach. Journal of Sports Engineering and Technology. 2015;229(1): 3-9.

[16] Zolfagharian A, Lakhi M, Ranjbar S, Bodaghi M. Custom shoe sole design and modeling toward 3D printing. International Journal of Bioprinting. 2021;7(4).

[17] Huang W. Insole Design Method Research Based on Foot Shape and Plantar Pressure Distribution. Huaqiao University.2016.

[18] Amorim D J N, Nachtigall T, Alonso M B. Exploring Mechanical Meta-Material Structures through Personalised Shoe Sole Design. Association for Computing Machinery. 2019;1-8. 